$1 N O 3$

NASA Technical Memorandum 110411

\title{
Conflict Probability Estimation for Free Flight
}

Russell A. Paielli and Heinz Erzberger

October 1996

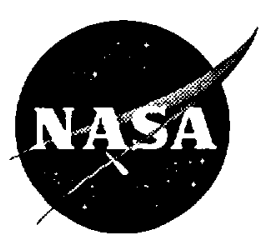

National Aeronautics and

Space Administration 


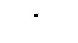


NASA Technical Memorandum 110411

\section{Conflict Probability Estimation for Free Flight}

Russell A. Paielli and Heinz Erzberger, Ames Research Center, Moffett Field, California

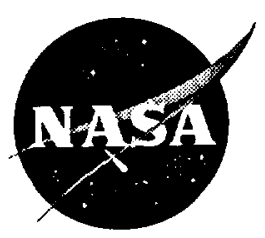

National Aeronautics and Space Administration

\section{Ames Research Center}

Moffett Field, California 94035-1000 



\title{
CONFLICT PROBABILITY ESTIMATION FOR FREE FLIGHT
}

\author{
Russell A. Paielli and Heinz Erzberger
}

Ames Research Center

\section{SUMMARY}

The safety and efficiency of free flight will benefit from automated conflict prediction and resolution advisories. Conflict prediction is based on trajectory prediction and is less certain the farther in advance the prediction, however. An estimate is therefore needed of the probability that a conflict will occur, given a pair of predicted trajectories and their levels of uncertainty. This paper presents a method to estimate that conflict probability. The trajectory prediction errors are modeled as normally distributed, and the two error covariances for an aircraft pair are combined into a single, equivalent covariance of the relative position. A coordinate transformation is then used to derive an analytical solution. Numerical examples and a Monte Carlo validation are presented.

\section{INTRODUCTION}

The economics and efficiency of air transportation in the continental U.S. could be improved significantly if the rigid routing restrictions currently imposed by the Federal Aviation Administration (FAA) (ref. 1) were relaxed to allow more direct or wind-optimal trajectories. The current routing restrictions help to maintain the safe and orderly flow of traffic, but new technologies are being developed to make them unnecessary. The ultimate goal is free flight (refs. 2 and 3), which could save the airlines several billion dollars per year in direct operating costs, according to the Air Transport Association (ATA). The safety and efficiency of free flight will benefit from automated conflict predictions and resolution advisories. By definition, a conflict (not to be confused with a collision) occurs when two or more aircraft come within the minimum allowed distance between each other. The minimum allowed horizontal separation for en-route airspace is currently 5 nautical miles (n.mi.). The vertical separation requirement above an altitude of 29,000 feet (ft) is currently $2000 \mathrm{ft}$; below that level it is $1000 \mathrm{ft}$.

Aircraft trajectory prediction is inexact, primarily because of wind modeling and prediction errors and secondarily because of tracking and flight-control errors. Wind estimates, based on the Mesoscale Analysis and Prediction System Rapid Update Cycle (MAPS/RUC) (refs. 4 and 5), are provided by the National Oceanic and Atmospheric Administration (NOAA). The farther in advance trajectories are predicted, the more uncertain those predictions are, particularly in the along-track direction, because aircraft in cruise are usually programmed to maintain a particular airspeed, and the resulting groundspeed depends on the winds. Because conflict prediction is based on trajectory prediction, the farther in advance a potential conflict is predicted to occur or not to occur, the less certain that prediction is likely to be. A method is needed to estimate the level of certainty.

The optimal time to initiate a conflict-resolution maneuver is a trade-off between efficiency and certainty. The farther in advance a maneuver is initiated. the more efficient it is likely to be in terms of extra distance flown, but the less certain will be exactly what maneuver is required or whether a maneuver is required at all. The later a maneuver is initiated, on the other hand, the more certain will be exactly what maneuver is required, but the less efficient and more harsh the maneuver is likely to be. The determination of the optimal time to initiate a maneuver, therefore. requires an esimate of conflict probability.

The determination of the optimal maneuver to be executed also requires a method of estimating conflict probability, because the goal of conflict resolution is to reduce the post-resolution conflict probability to some acceptable level. The conflict probability cannot be reduced to zero without introducing gross inefficiency, but that is not necessary because human air traffic controllers will be available to catch any unresolved 
conflicts. The methods presented in this paper are intended to assist rather than replace human air traffic controllers. That is, they are intended to provide automated advisories for the controllers, but not to make the ultimate decisions.

A method is developed in this paper to estimate the conflict probability for a pair of aircraft in free flight. The trajectory prediction errors are modeled as normally distributed, and the two error covariances for an aircraft pair are combined into a single, equivalent covariance of the relative position. A coordinate transformation is used to derive an analytical solution. The paper is organized as follows: first, some background is given on modeling of trajectory prediction errors and conflict prediction; the conflict probability estimation algorithm is then developed. Finally, some numerical examples and a Monte Carlo validation are presented.

\section{CONFLICT PREDICTION}

Conflict prediction can be divided into the following three steps: First, the trajectories of all aircraft in the region of interest are predicted for approximately the next 20 to 30 minutes ( $\mathrm{min}$ ). These deterministic predictions are based on current estimated positions and velocities, flight plans, and predicted winds aloft. This complex modeling and software problem has already been solved for arrival traffic (ref. 6), and that solution will be adapted for en-route and departure traffic also. The second step is to coarsely screen all possible aircraft pairs to eliminate those with a negligible possibility of conflict. The third step, which is the subject of this paper, is to estimate the conflict probability for those remaining aircraft pairs. This probability involves the predicted trajectories and an estimate of their uncertainty.

In aircraft equipped with a Flight Management System (FMS), the lateral feedback loop is typically closed around cross-track position, and the stabilized cross-track rms (root mean square) prediction error is approximately constant. Typical magnitudes are from less than $0.5 \mathrm{n} . \mathrm{mi}$. to more than $1 \mathrm{n} . \mathrm{mi}$. Longitudinal position control, on the other hand, involves using the throttle to compensate for unpredictable variations in headwind or tailwind. This compensation tends to be inefficient in cruise, so the longitudinal feedback loop is usually closed around Mach number or airspeed, but not groundspeed or along-track position. For trajectory predictions of up to 20 or $30 \mathrm{~min}$, the unstabilized along-track rms error tends to grow approximately linearly, primarily because of wind-prediction bias error, as illustrated in figure 1. (This linear growth is typical but is not assumed or required by the algorithm presented in this paper.) A typical growth rate is $0.25 \mathrm{n} . \mathrm{mi} . / \mathrm{min}$ ( $15 \mathrm{knots} / \mathrm{min}$ ) in cruise (ref. 7 ), but this rate could be reduced in the future with improved wind modeling (ref. 8); it is greater in climb and descent.

Trajectory prediction errors based on live air traffic data were analyzed in reference 7 and found to be approximately normally distributed or Gaussian. The corresponding error ellipses tend to have their major principal axis in the along-track direction and their minor principal axis in the cross-track direction. (Note that the uncertainty ellipse for a normally distributed random variable $x$ is defined as the solution of $z^{T} Z^{-1} z=c^{2}$, where $z=x-E(x)$, $Z=\operatorname{cov}(z) \equiv E\left(z z^{T}\right), E$ is the expected value, and $c$ is a constant that can be assumed to be unity unless otherwise noted.)

The cross correlation of prediction errors between aircraft can also be important because common errors cancel in the position difference or relative position.

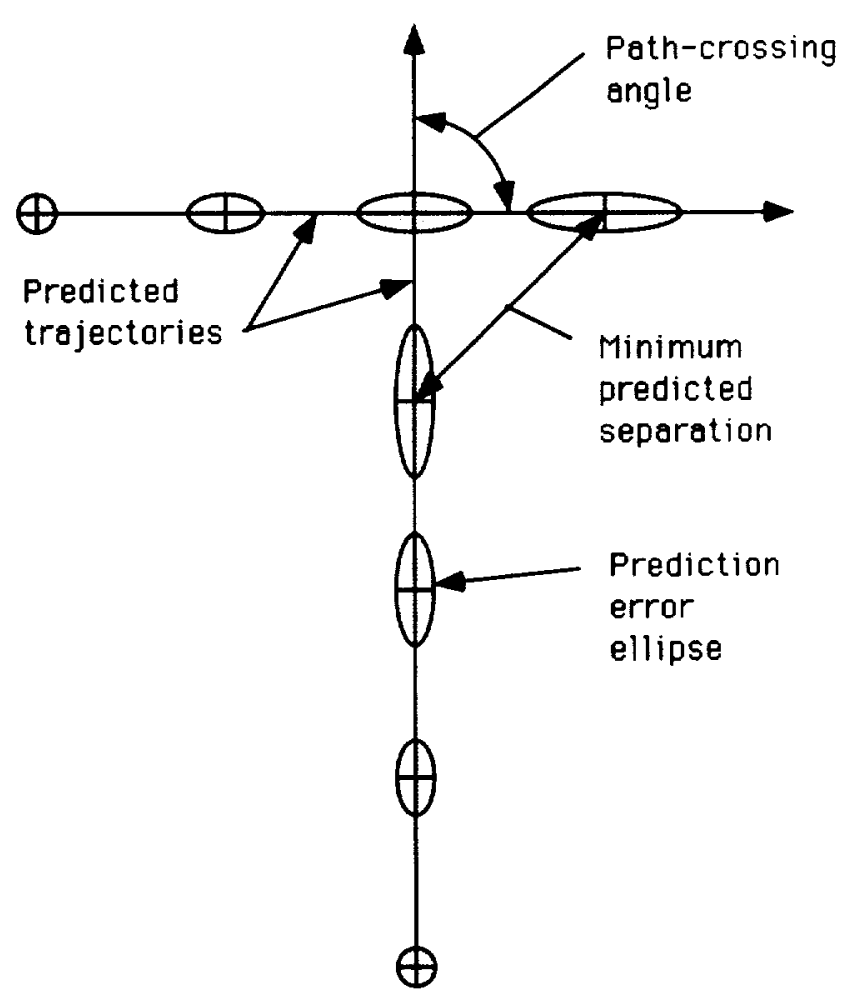

Figure 1. Trajectory prediction error ellipses. 
Unfortunately, the cross correlation is more difficult to model than the individual covariances because it depends on the trajectories and a spatial wind-error correlation model. The spatial wind-error correlation model will be a function of both separation distance and heading angular difference. Aircraft pairs with nearly perpendicular flight paths will tend to have weakly crosscorrelated prediction errors because their along-track positions are affected by different wind components. Aircraft pairs with small path-crossing angles and small minimum separations, on the other hand, will tend to have more strongly cross-correlated prediction errors, both because they are affected by a common wind component and because they spend a relatively long time close together. Although this area is open for research, it will not be pursued in this paper.

\section{CONFLICT PROBABILITY ESTIMATION}

This section is divided into five subsections. First, the method of combining two prediction-error covariances into a single covariance of the relative position is discussed. Next, a coordinate transformation is proposed that transforms the combined error covariance into a standard form. Then the analytical solution for the conflict probability in two dimensions is developed. The generalization from two to three dimensions is then discussed. Finally, the application to conflict resolution is previewed.

\section{Combined Error Covariance}

The trajectory prediction error for an aircraft will be modeled as normally distributed, with zero mean and with a covariance that has eigenvectors in the along-track and cross-track directions, as explained previously. The covariance matrix is therefore diagonal in a coordinate system aligned with the aircraft heading. If $q$ is the aircraft position in such a headingaligned coordinate system, and $\bar{q}$ is the corresponding prediction, then the prediction error is

$$
\tilde{q} \equiv q-\bar{q}
$$

and the corresponding diagonal covariance matrix is

$$
S \equiv \operatorname{cov}(\tilde{q})
$$

where $\operatorname{cov}(x) \equiv E\left(x x^{T}\right)$ for any random variable $x$, and $E$ is the expected value function. If $\psi$ is the heading angle in some Earth-fixed reference coordinate system, then

$$
R \equiv\left[\begin{array}{rr}
\cos \psi & -\sin \psi \\
\sin \psi & \cos \psi
\end{array}\right]
$$

is a rotation matrix that transforms the heading-aligned coordinates to the reference coordinates. The position prediction error in the reference coordinate system is then

$$
\tilde{p}=R \tilde{q}
$$

and the corresponding covariance matrix is

$$
Q \equiv \operatorname{cov}(\tilde{p})=R S R^{T}
$$

Because the trajectory prediction errors are modeled as normally distributed, the two error covariances for an aircraft pair can be easily combined into a single equivalent covariance of the position difference or the relative position of one aircraft with respect to the other. For present purposes, this combined covariance can be assigned to one of the aircraft, referred to as the "stochastic" aircraft, and the other aircraft, referred to as the "reference" aircraft, can be regarded as having no position uncertainty.

Let subscripts $S$ and $R$ designate the stochastic and reference aircraft, respectively. The position difference is

$$
\Delta p \equiv p_{S}-p_{R}
$$

The prediction of that position difference is

$$
\Delta \bar{p} \equiv \bar{p}_{S}-\bar{p}_{R}
$$

and the prediction error is

$$
\Delta \tilde{p} \equiv \Delta p-\Delta \bar{p}=\tilde{p}_{S}-\tilde{p}_{R}
$$

The combined prediction error covariance is then

$$
M \equiv \operatorname{cov}(\Delta \tilde{p})=Q_{S}+Q_{R}-Q_{S R}
$$

where $Q_{S}$ and $Q_{R}$ are the individual covariances based on equation (5), and the cross-correlation term $Q_{S R}$ is defined as

$$
Q_{S R} \equiv E\left(\tilde{p}_{S} \tilde{p}_{R}^{T}+\tilde{p}_{R} \tilde{p}_{S}^{T}\right)
$$

In general, the error ellipse corresponding to the combined error covariance will no longer have principal axes aligned with along-track and cross-track directions of either aircraft. 
Figure 2 shows an example encounter geometry, with the combined error ellipse centered on the stochastic aircraft, and the circular conflict zone (5-n.mi. radius) centered on the reference aircraft. The error ellipse corresponds to a probability density function that can be represented as a surface over the ellipse. The ellipse is actually the intersection of that surface and a horizontal plane cutting the surface. The total volume under the surface is unity. The probability of conflict at a particular time is the portion of that volume that is within the circular conflict zone. An analytical solution has not been found for this probability, but it is not as important as the total probability of conflict for the encounter, which is discussed in the following paragraphs.

It is assumed that the aircraft velocities and prediction errors are constant during the period of potential conflict, which is at least approximately true for most aircraft pairs in free flight. The total probability of conflict for the encounter can then be determined as follows: Project the circular conflict zone along a line parallel to the relative velocity to form an extended conflict zone, as illustrated in figure 2 . The conflict probability is equal to the portion of the volume under the probability density surface that is within this extended conflict zone. The coordinate transformation to

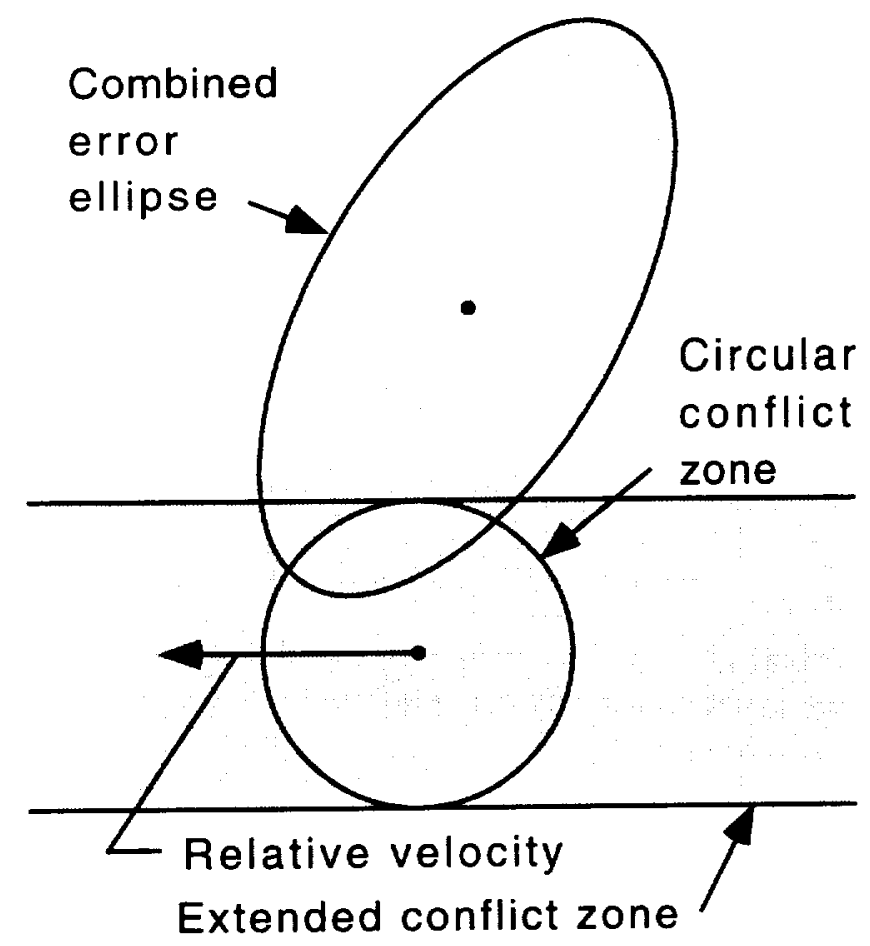

Figure 2. Encounter geometry. be presented in the next section allows this probability to be determined analytically.

\section{Coordinate Transformation}

Coordinate transformations are often useful for simplifying problems. They are widely used in control theory, for example. In this case, the conflict probability is difficult or impossible to determine analytically in the original coordinate system. It can be determined numerically, but a numerical solution is likely to be much less efficient and less accurate than an analytical solution. This inefficiency is undesirable for an algorithm that is intended to run in real time at a very high rate for many years. Fortunately, a coordinate transformation has been found that allows an analytical solution.

Let $p$ and $\rho$ represent the original and transformed coordinates of position, respectively. A general linear coordinate transformation is of the form

$$
\begin{gathered}
\rho=T p \\
p=W \rho
\end{gathered}
$$

where $T$ is a transformation matrix to be determined, and $W \equiv T^{-1}$. The transformations for velocity and other vectors are of the same form. Combining the definition

$$
\Delta \rho \equiv \rho_{S}-\rho_{R}
$$

with definitions (6)-(8) gives

$$
\Delta \tilde{\rho}=T \Delta \tilde{p}
$$

In the transformed coordinate system, the mean prediction error is still zero and the combined error covariance is

$$
\operatorname{cov}(\Delta \tilde{\rho})=T M T^{T}
$$

where $M \equiv \operatorname{cov}(\Delta \tilde{p})$ is the combined error covariance in the original coordinate system from equation (9).

A Cholesky decomposition (ref. 9) or "squareroot" factorization of the combined error covariance $M$ is of the form

$$
M=L L^{T}
$$

where $L$ is lower triangular. If $T$ is of the form

$$
T=R L^{-1}
$$


where $R$ is any orthogonal rotation matrix, then equation (15) becomes

$$
\operatorname{cov}(\Delta \tilde{\rho})=I
$$

where the fact that $R R^{T}=I$ has been used. The combined error ellipse is therefore in the standard form of a unit circle, as shown in figure 3 . The conflict boundary, which was a circle in the original coordinate system, is an ellipse in the transformed coordinate system, also as shown in figure 3 .

\section{Analytical Solution}

Having the error ellipse in the form of a unit circle simplifies the probability computation considerably because the corresponding two-dimensional (2-D) probability density function decouples into the product of two identical one-dimensional (1-D) functions: $p(x, y)=p(x) p(y)$, where $p(x)=\exp \left(-x^{2} / 2\right) / \sqrt{2 \pi}$. The probability density function can be represented as a radially symmetric surface over the circle. The circle is actually the intersection of that surface and a horizontal plane cutting the surface. The total volume under the surface is unity.

In the transformed coordinate system, the extended conflict zone is still in the direction of the (transformed) relative velocity, and the conflict probability is still equal to the portion of the volume under

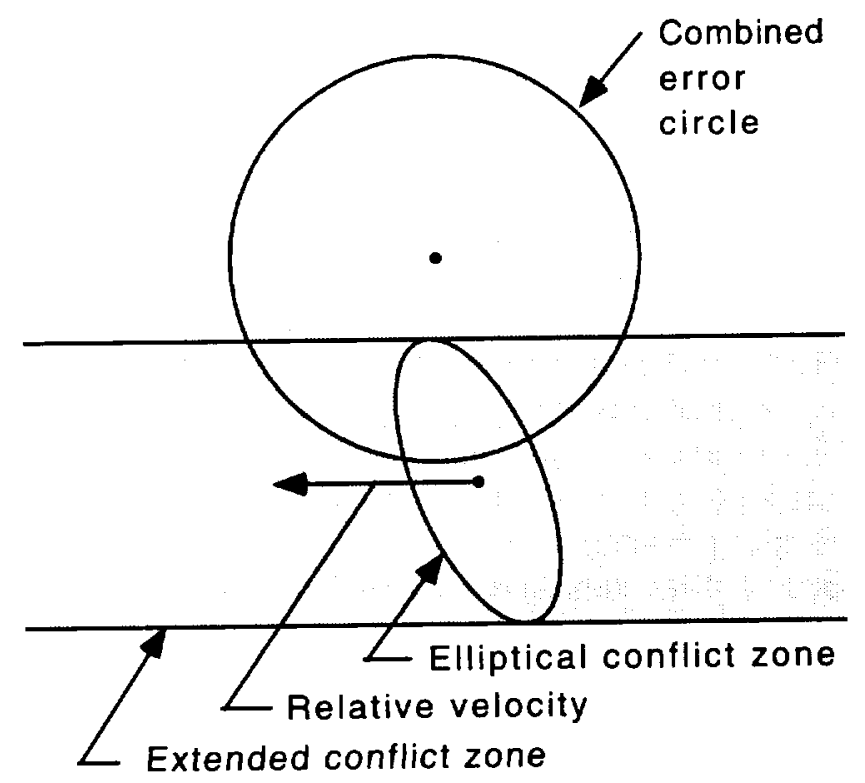

Figure 3. Transformed encounter geometry. the probability density surface that is within this extended conflict zone. The rotation matrix $R$ in equation (17) can be used to rotate the transformed coordinate system about the origin. It can therefore be selected such that the relative velocity is in the positive or negative $x$-direction. If $\Delta v \equiv v_{S}-v_{R}$ is the relative velocity in the original coordinate system, and $\Delta \nu \equiv\left(\Delta \nu_{x}, \Delta \nu_{y}\right) \equiv L^{-1} \Delta v$ is the partially transformed relative velocity, then

$$
R=\frac{1}{\|\Delta \nu\|}\left[\begin{array}{rr}
\Delta \nu_{x} & \Delta \nu_{y} \\
-\Delta \nu_{y} & \Delta \nu_{x}
\end{array}\right]
$$

The boundaries of the extended conflict zone are then the minimum and maximum values of $y$ on the elliptical conflict boundary.

Let $\Delta p_{c}$ and $\Delta \rho_{c}$ represent the original and transformed coordinates, respectively, of points on the conflict boundary relative to the reference aircraft. The equation of the conflict boundary is

$$
\left\|\Delta p_{c}\right\|=\left\|W \Delta \rho_{c}\right\|=s_{c}
$$

where $s_{c}$ is the conflict separation distance ( $5 \mathrm{n} . \mathrm{mi}$.) and $W$ is defined in equation (12). This equation can be squared, then expanded according to

$$
\Delta \rho_{c} \equiv\left[\begin{array}{c}
\Delta x_{c} \\
\Delta y_{c}
\end{array}\right], \quad W^{T} W \equiv\left[\begin{array}{cc}
a & b \\
b & c
\end{array}\right]
$$

The resulting equation for the elliptical conflict boundary is

$$
a \Delta x_{c}^{2}+2 b \Delta x_{c} \Delta y_{c}+c \Delta y_{c}^{2}=s_{c}^{2}
$$

The minimum and maximum values of $\Delta y_{c}$ can then be determined by at least two different methods. One method is to consider equation (22) as a quadratic equation in $\Delta x_{c}$ with coefficients that are functions of $\Delta y_{c}$. The minimum and maximum values of $\Delta y_{c}$ can then be determined by setting the discriminant of that quadratic equation to zero and solving for $\Delta y_{c}$. Another method is to differentiate equation (22) with respect to $\Delta x_{c}$ and solve the equation $d\left(\Delta y_{c}\right) / d\left(\Delta x_{c}\right)=0$, together with equation (22). The result is

$$
\Delta y_{c}= \pm s_{c} \sqrt{a /\left(a c-b^{2}\right)}
$$

at the minimum and maximum points. Note that $a$ is positive and $a c-b^{2}$ is positive and invariant with respect to rotation for any ellipse (ref. 10), so the argument of the square root function must also be positive. 
The conflict probability is the portion of the volume under the surface of the probability density function that is within the extended conflict zone. Because the probability density function decouples into $p(x, y)=p(x) p(y)$ and the conflict boundaries are parallel to the $x$-axis, the expression for the conflict probability $P_{c}$ can be simplified as follows:

$$
\begin{aligned}
P_{c} & =\int_{-\Delta y-\Delta y_{c}}^{-\Delta y+\Delta y_{c}} \int_{-\infty}^{\infty} p(x, y) d x d y \\
& =\int_{-\Delta y-\Delta y_{c}}^{-\Delta y+\Delta y_{c}} p(y) d y \int_{-\infty}^{\infty} p(x) d x \\
& =\int_{-\Delta y-\Delta y_{c}}^{-\Delta y+\Delta y_{c}} p(y) d y \\
& =P\left(-\Delta y+\Delta y_{c}\right)-P\left(-\Delta y-\Delta y_{c}\right)
\end{aligned}
$$

where $\Delta y \equiv y_{S}-y_{R}$ is the $y$-coordinate of the stochastic aircraft with respect to the reference aircraft, and $P$ is the cumulative normal probability function. The latter, defined such that $P(z) \equiv \int_{-\infty}^{z} p(s) d s$ for any random variable $z$, can be determined analytically (ref. 9). This analytical solution for the conflict probability is therefore theoretically exact under the assumptions stated previously.

The main assumption is that the aircraft velocities are constant (in both magnitude and direction) during the period of potential conflict. Free-flight trajectories typically will be fairly direct and have few turns, so that assumption is likely to be accurate in most cases. For constant velocity, the time at which the minimum predicted separation occurs is

$$
t_{m}=t_{0}+\frac{\Delta p_{0}^{T} \Delta v}{\Delta v^{T} \Delta v}
$$

where $\Delta p_{0}$ is the position difference at time $t_{0}$, and $\Delta v$ is the constant velocity difference, both in terms of cartesian coordinates. The position difference at minimum separation is then

$$
\Delta p_{m}=\Delta p_{0}+\left(t_{m}-t_{0}\right) \Delta v
$$

The minumum separation distance itself is $\left\|\Delta p_{m}\right\|$.

Small variations in aircraft velocity due to wind disturbances or wind-optimal routing have only a small effect in the immediate vicinity of an encounter, so they will not significantly violate the assumption of constant velocity. The predicted velocities at the point of minimum predicted separation are tangent to the flightpaths and can be considered first-order linear approximations to the actual trajectories at that point. In the unlikely case that a large heading or speed change is scheduled in the vicinity of a potential conflict, on the other hand, the analytical solution for conflict probability will not be accurate.

\section{Three-Dimensional Case}

If the two aircraft are in level flight at different altitudes, or if one or both of the aircraft are climbing or descending, the problem is three-dimensional (3-D). The basic modifications required to the 2-D case are discussed in this section. For simplicity, the alongtrack axis is defined as the projection of the predicted velocity vector on a horizontal plane. The along-track and cross-track axes are therefore horizontal by definition, and the prediction error ellipsoid is modeled as having its principal axes in the along-track, cross-track, and vertical directions. For en-route flight, the conflict zone is a cylinder or disk with a horizontal radius of 5 n.mi. and a vertical thickness of $2000 \mathrm{ft}$.

A coordinate transformation can be used to transform the error ellipsoid into a unit sphere. Most of the previous analysis still applies, but in three dimensions rather than two. The transformation can be decoupled into a 2-D horizontal transformation identical to the one discussed previously and a vertical transformation that is a simple scaling. The conflict zone, which is a circular cylinder in the original coordinate system, is an elliptical cylinder in the transformed coordinate system. The transformation can still be selected such that the relative velocity is in the positive or negative $x$-direction.

Consider first the case in which both aircraft are in level flight, but at different altitudes. In this case the relative velocity vector is horizontal, and the projection of the disc-shaped conflict zone along the direction of relative velocity forms a rectangular volume. The conflict probability is the product of two cumulative normal probability differences, one that is identical to equation (24), and another of the same form that applies to the vertical axis. That is, the horizontal conflict probability of equation (24) can be generalized to three dimensions by multiplying it by a vertical conflict-probability factor. The vertical factor is $P\left(-\Delta z+\Delta z_{c}\right)-P\left(-\Delta z-\Delta z_{c}\right)$, where $\Delta z$ is the predicted vertical separation between the two aircraft, and $\Delta z_{c}$ is the minimum allowed vertical separation ( $2000 \mathrm{ft}$ ), both normalized (divided) by the vertical $\mathrm{rms}$ error. 
A typical vertical rms error, which is caused primarily by baro-altimeter error, is approximately $100 \mathrm{ft}$. For all practical purposes, it can be assumed that the vertical error will not exceed $\pm 400 \mathrm{ft}$ for each aircraft or $\sqrt{2} \times 400 \approx 600 \mathrm{ft}$ for the altitude difference of two aircraft. Therefore, if the predicted vertical separation is less than about $2000-600=1400 \mathrm{ft}$, the vertical factor is virtually unity, and the 3-D conflict probability is essentially equal to the horizontal conflict probability. If the predicted vertical separation is greater than about $2000+600=2600 \mathrm{ft}$, on the other hand, the vertical factor is virtually zero, and the horizontal conflict probability need not even be computed. For a vertical rms error of $100 \mathrm{ft}$, therefore, the vertical factor needs to be computed only if the predicted vertical separation is between about 1400 and $2600 \mathrm{ft}$.

The case in which one or both of the aircraft are climbing or descending is more complicated, unfortunately, because the relative velocity is not horizontal, and the projection of the disc-shaped conflict zone along the direction of relative velocity does not form a rectangular volume. The cross section of that volume is a rectangle with halves of an ellipse attached to the top and bottom. Numerical integration can be used to approximate the conflict probability, if necessary, or some heuristic approximation may be possible, but that approximation will not be pursued here.

\section{Application to Conflict Resolution}

The ultimate purpose of conflict-probability estimation is for use in optimal conflict resolution. The problem of conflict resolution involves deciding when to initiate a resolution maneuver and what maneuver to execute. The conflict probability is an important factor in both decisions. This subsection outlines horizontal conflict-resolution methods presently under investigation. Vertical conflict resolution will also be discussed briefly.

The optimal time to initiate a conflict-resolution maneuver is a trade-off between efficiency and certainty. The farther in advance a maneuver is initiated, the more efficient it is likely to be in terms of extra distance flown, but the less certain will be exactly what maneuver is required or whether a maneuver is required at all. The later a maneuver is initiated, on the other hand, the more certain will be exactly what maneuver is required, but the less efficient and more harsh the maneuver is likely to be. The optimal time to initiate a maneuver can be determined by minimizing a cost function that reflects the statistically expected cost of maneuvering (or not maneuvering) as a function of time. That cost function depends on the conflict probability, the operating cost per unit of distance traveled, and various other issues such as passenger comfort and controller workload.

A conflict is resolved in the horizontal plane by moving the extended conflict zone sufficiently far away from the center of the error ellipse or circle to reduce

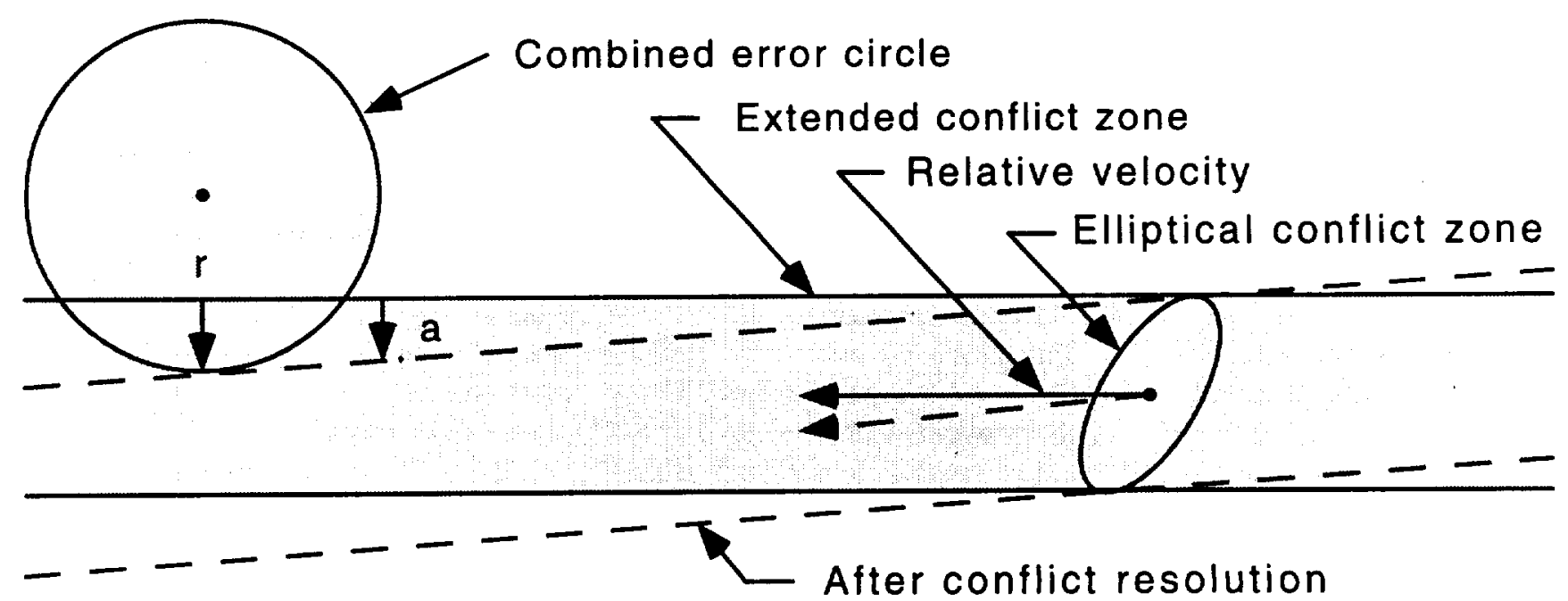

Figure 4. Conflict resolution geometry in transformed coordinate system. 
the conflict probability to some desired level. The resolution maneuver involves changing the direction of the relative velocity for some period of time, as illustrated in figure 4 . The magnitude of the relative velocity is essentially irrelevant. It is assumed that the aircraft will complete the dynamic or accelerating portion of the maneuver and reach a constant velocity before the encounter (period of potential conflict). The velocity changes can therefore be modeled as instantaneous until the static maneuver is determined; then the dynamic transients can be properly accounted for.

The key parameters are the time at which the maneuver is initiated, the time at which it is completed, and the angular change of the relative velocity. If the maneuver is completed after the encounter, as shown in figure 4 , its effect is to rotate the extended conflict zone by an angle $a$ about the point at which the maneuver is initiated. If it is completed before the encounter, on the other hand, and the aircraft return to their original velocities, its effect is to translate the extended conflict zone by a distance $r$ perpendicular to the relative velocity.

The direction of the relative velocity after resolution can be easily transformed back to the original coordinate system. Then, changes in the individual aircraft velocities must be determined to realize that relative velocity. In general, the change in relative velocity can involve changes in both the magnitude and the direction of the individual velocities. The solution is underdetermined, however, and additional constraints can be applied to simplify the maneuver. For example, the solution can be constrained to require only one aircraft to maneuver. In addition, the maneuver can be further constrained to consist of only a heading change at constant speed or a speed change at constant heading, if desired.

This discussion applies mainly to horizontal confict resolution, but vertical conflict resolution is also very important for two reasons. First, because the minimum required separation is about 15 times less in the vertical axis than it is in the horizontal plane, vertical conflict resolution may be more efficient in many cases, particularly when the minimum predicted horizontal separation is small and/or the vertical separation is already almost large enough to avoid a conflict. Second, because vertical conflict resolution is much simpler, it may be appropriate for conflicts involving three or more aircraft. For those cases, determination of efficient horizontal resolution maneuvers can be mathematically complicated and computationally intensive, and accurate and reliable execution can be operationally difficult. Separating the aircraft by altitude, on the other hand, is much simpler.

\section{VALIDATION}

The Gaussian statistical model on which the conflict-probability algorithm is based was determined empirically by analyzing actual air traffic data (ref. 7). A Monte Carlo simulation was used to validate the algorithm itself. In the Monte Carlo simulation, combinations of path-crossing angles, minimum predicted separations, and times to minimum predicted separation were generated. For each combination, the conflict probability was computed and nominal trajectories were generated. Then the nominal trajectories were perturbed by a series of random prediction errors, each consisting of constant cross-track position error and constant along-track velocity error. Finally, the empirical fraction of cases in which conflicts resulted was compared with the computed conflict probability.

Table 1 shows a representative sampling of the differences between the computed conflict probabilities and the Monte Carlo simulation results. One million Monte Carlo samples were run for each entry in the table, and each entry corresponds to a particular encounter geometry. The algorithm matches well with the simulation results. The largest magnitude of the difference for all cases shown is 1.8 percent, and only 5 of the 72 differences in the table are at or over 1 percent in magnitude. Most of the differences are well under 1 percent in magnitude, and many are at about 0.1 percent. Given the accuracy of the underlying error model and the requirements of the application. this result is more than adequate. A worst-case accuracy of perhaps 5 percent would have been considered adequate.

The differences are larger than would be statistically expected, however. The expected standard deviation for each table entry is $\sqrt{P_{c}\left(1-P_{c}\right) / N}$, where $N$ is the number of samples and $P_{C}$ is the conflict probability. Note that $P_{c}\left(1-P_{c}\right)=0$ if $P_{c}=0$ or $P_{c}=1$, and the maximum of $\sqrt{P_{c}\left(1-P_{c}\right)}$ is 0.5 when $P_{c}=0.5$. Thus, the maximum expected standard deviation for any table entry cannot exceed 0.0005 . One reason that the differences are larger than expected is that the analytical solution is based on the 
Table 1. Monte Carlo simulation results: computed minus empirical conflict probability based on 1,000,000 samples per entry

\begin{tabular}{|c|r|rrrr|}
\hline \multicolumn{6}{|l}{ Path-crossing angle, deg } \\
\cline { 2 - 6 } & \multicolumn{4}{ll}{ Minimum predicted separation, n.mi. } \\
\cline { 3 - 6 } & & \multicolumn{4}{l|}{ Time to minimum separation, min } \\
\cline { 3 - 6 } & & 4 & 8 & 12 & 24 \\
\hline \hline 15 & 0 & +0.000 & -0.009 & -0.006 & -0.002 \\
15 & 5 & -0.018 & -0.003 & -0.003 & -0.002 \\
15 & 10 & -0.001 & -0.006 & -0.005 & -0.003 \\
\hline 30 & 0 & +0.001 & +0.006 & +0.005 & +0.001 \\
30 & 5 & -0.011 & -0.004 & -0.001 & +0.000 \\
30 & 10 & -0.000 & -0.004 & -0.004 & -0.001 \\
\hline 45 & 0 & +0.002 & +0.012 & +0.010 & +0.003 \\
45 & 5 & -0.006 & -0.003 & -0.000 & +0.002 \\
45 & 10 & -0.001 & -0.006 & -0.006 & -0.001 \\
\hline 90 & 0 & -0.000 & -0.000 & +0.000 & +0.001 \\
90 & 5 & -0.001 & -0.001 & -0.000 & -0.000 \\
90 & 10 & -0.000 & -0.000 & -0.000 & +0.000 \\
\hline 135 & 0 & -0.000 & -0.003 & -0.008 & -0.012 \\
135 & 5 & +0.001 & -0.001 & +0.000 & -0.000 \\
135 & 10 & +0.000 & +0.001 & +0.004 & +0.006 \\
\hline 180 & 0 & +0.000 & +0.000 & +0.000 & +0.000 \\
180 & 5 & -0.000 & -0.000 & +0.000 & -0.001 \\
180 & 10 & -0.000 & -0.000 & -0.000 & -0.000 \\
\hline
\end{tabular}

assumption that the prediction error covariance is constant during the encounter, whereas it actually grows with prediction time.

\section{NUMERICAL EXAMPLES}

A set of numerical examples of conflict probabilities and related quantities were generated as a function of encounter geometry. The aircraft speeds were $8 \mathrm{n} . \mathrm{mi} . / \mathrm{min}$ (480 knots) in every case, a typical speed for commercial transport aircraft. The conflict separation distance was $5 \mathrm{n} . \mathrm{mi}$., the currently used value for en-route airspace. The cross-track rms error was 1 n.mi., and the along-track rms error started at zero and grew linearly at a rate of $0.25 \mathrm{n} . \mathrm{mi} . / \mathrm{min}$, unless otherwise stated. These values are typical for cruise. (This linear-growth model is typical but is not assumed or required by the algorithm.) Wind-error cross correlation between aircraft was not modeled.

Figure 5 shows the cumulative separation probability, with prediction time as a parameter, where the

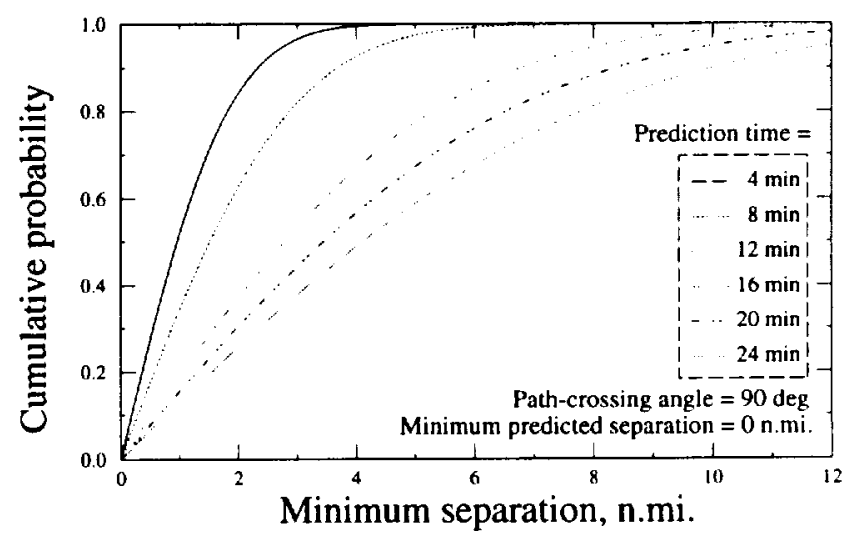

Figure 5. Cumulative separation probability.

path-crossing angle is $90 \mathrm{deg}$ and the minimum predicted separation is zero n.mi. (an exact collision). This value is the probability that the minimum separation will be less than the abscissa value. Figure 6 shows the corresponding probability density (the derivative, with respect to minimum separation, of the cumulative separation probability shown in figure 5 , determined by numerical differentiation). This plot 


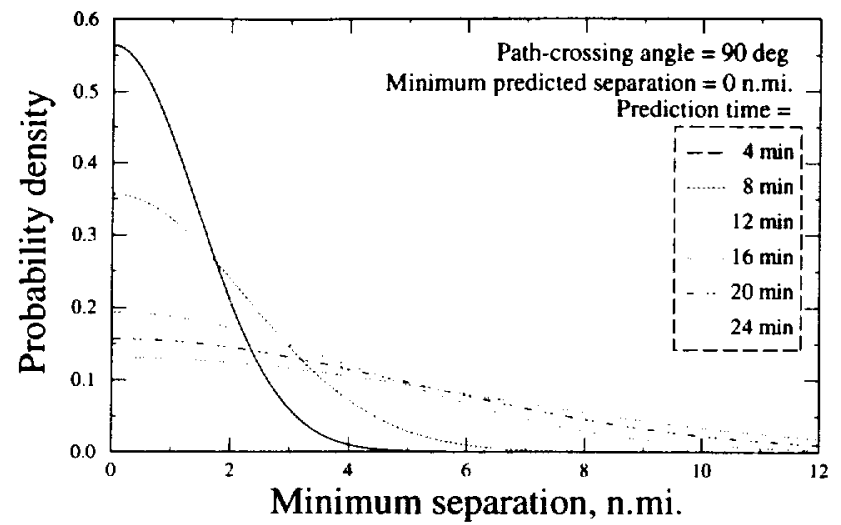

Figure 6. Separation probability density.

shows how the density function spreads out as prediction time increases.

It is interesting to see how the expected value of the minimum separation compares with the predicted value as prediction time increases. The predicted value is based on a deterministic trajectory model, with no consideration for trajectory-prediction errors. The expected or mean value, on the other hand, is derived from the cumulative separation probability function $P$ or the separation probability density function $p$ according to $E(s)=\int_{0}^{\infty}[1-P(x)] d x=\int_{0}^{\infty} x p(x) d x$. Figure 7 shows the expected value of minimum separation as a function of time to minimum predicted separation, with minimum predicted separation as a parameter, where the path-crossing angle is $90 \mathrm{deg}$. The expected separation diverges from the predicted separation, but the group of curves shown converge to a common asymptote for large prediction times. Note that the 0 n.mi. case does not intersect the origin because the cross-track rms error is nonzero at time zero.

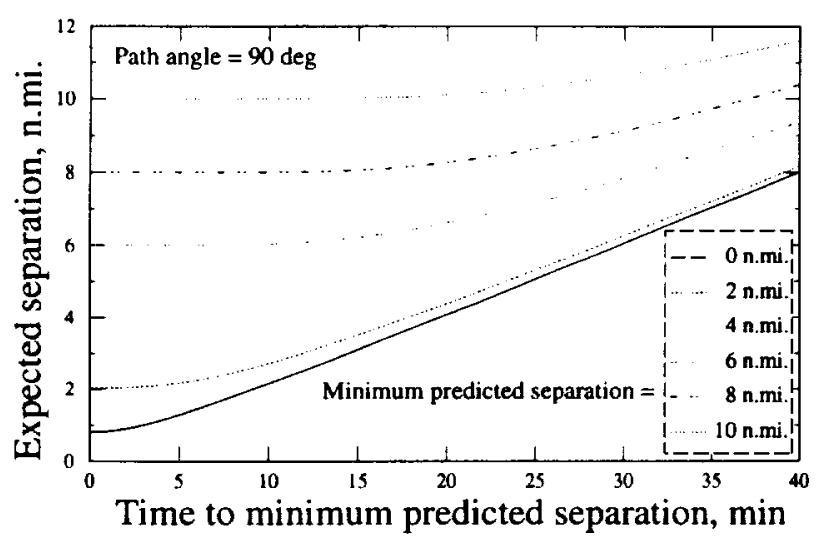

Figure 7. Expected value of minimum separation.

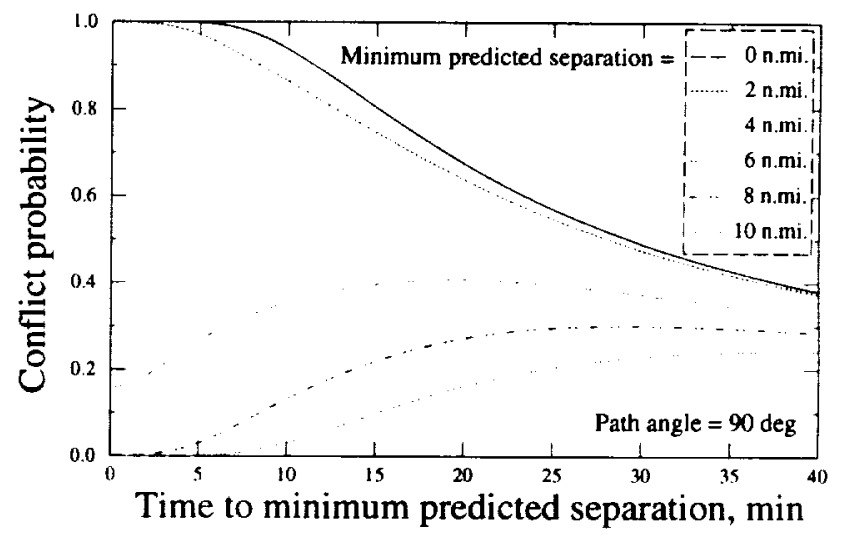

Figure 8. Effect of minimum predicted separation.

Figure 8 shows the effect of minimum predicted separation on conflict probability. Conflict probability is plotted as a function of the time to minimum predicted separation, with the minimum predicted separation as a parameter, where the path-crossing angle is $90 \mathrm{deg}$. For small prediction times, the covariances are small and the conflict probabilities are a strong function of minimum predicted separation. For larger prediction times, the covariances grow and the conflict probability becomes a weaker function of the minimum predicted separation. The conflict probabilities converge and asymptotically approach zero as prediction time increases.

Figure 9 shows the effect of path-crossing angle on conflict probability. Conflict probability is plotted again as a function of the time to minimum predicted separation, but with the path-crossing angle as a parameter, where the predicted minimum separation is 0 n.mi. As a point of reference, the curve for the path-crossing angle of $90 \mathrm{deg}$ is a repeat of the

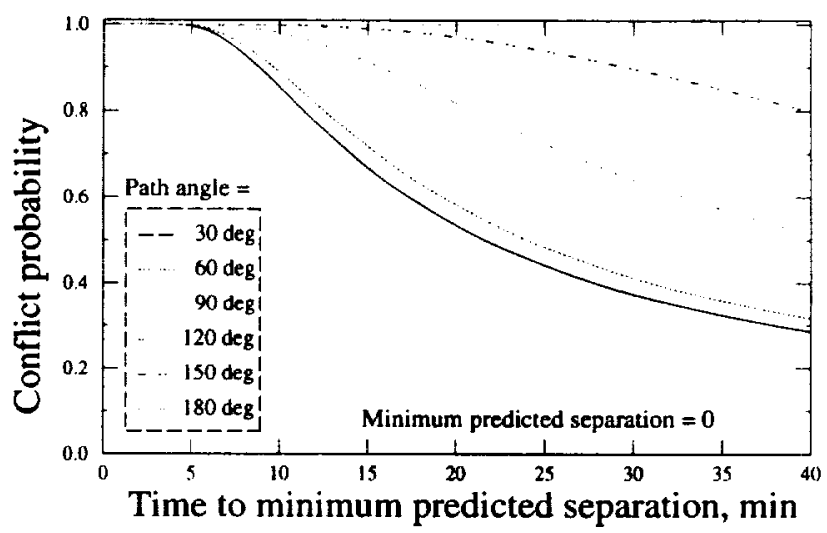

Figure 9. Effect of path-crossing angle. 
corresponding curve of figure 8 . As the prediction time increases, the conflict probability decreases faster for smaller path-crossing angles. If wind-error cross correlation were taken into account, however, these curves would be very different for smaller path-crossing angles. A portion of the trajectory-prediction error would cancel in the position difference, and the effective error growth rate would be smaller. Hence the conflict probabilities for smaller path angles would be higher than these shown in figure 9.

Figure 10 shows the effect of prediction-error growth rate on conflict probability. Conflict probability is plotted again as a function of the time to minimum predicted separation, but with the along-track rms error growth rate as a parameter. The value of $0.25 \mathrm{n} . \mathrm{mi} . / \mathrm{min}$ or $15 \mathrm{knots}$ has been used throughout this paper for the along-track rms error growth rate, but values of 10 knots and 20 knots are also shown in the figure. For each of the three values of error growth rate, conflict probability is plotted for minimum predicted separations of 0 and $10 \mathrm{n} . \mathrm{mi}$. Note that the three pairs of curves could be collapsed into one pair by scaling the horizontal axis by the along-track error growth rate. That is, if the horizontal axis were the along-track error, the three pairs of curves would be identical. Showing them separately does, however, illustrate some important characteristics.

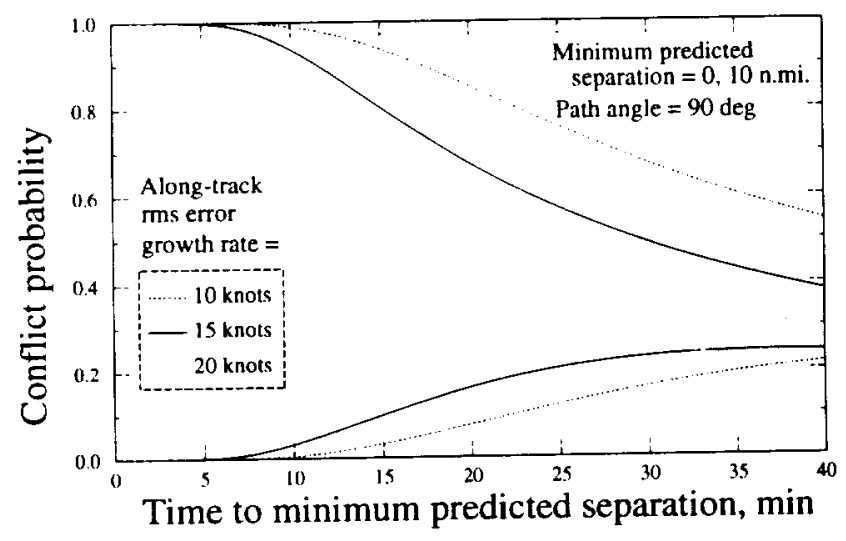

Figure 10. Effect of prediction-error growth rate.
For minimum predicted separations substantially less than the minimum allowed separation, the conflict probability starts at unity and decreases monotonically as a function of prediction time. The effect of larger error growth rates is to cause the conflict probability to decrease more rapidly as a function of prediction time. For minimum predicted separations substantially greater than the minimum allowed separation, on the other hand, the conflict probability starts at zero, increases to some maximum value, and then decreases back toward zero. This phenomenon occurs because the individual error ellipses expand and start to overlap, but then after the maximum conflict probability they expand even more and the probability density function becomes flatter. The effect of larger error growth rates is to cause the conflict probability to initially increase more rapidly as a function of prediction time, and then to decrease more rapidly after the maximum.

Finally, since computational efficiency is a major concern in a real-time air traffic control system, basic timing tests were performed on the conflict probability algorithm running on a Sun SPARC 10 workstation. These tests were for the conflict-probability algorithm only and did not include trajectory prediction, winderror modeling, or any other part of the problem. The average computation time per aircraft pair was slightly under 0.6 milliseconds. In addition to being theoretically exact under the stated assumptions, this time is one to four orders of magnitude faster than a numerical solution, depending on the method and level of resolution of the numerical integration. Furthermore, it is fast enough to be used directly in a real-time system.

\section{CONCLUSION}

A method is established to accurately and efficiently estimate the probability of conflict for aircraft pairs in free flight. Accurate probability estimates are necessary for optimal conflict resolution. The analysis behind the estimates, furthermore, is also very useful for developing an optimal conflict-resolution algorithm, which is presently under way. These methods can eventually be applied to actual air traffic to help controllers maintain safe and efficient free flight. 


\section{REFERENCES}

1. Federal Aviation Administration Handbook 7110.65J, Oct. 18, 1984.

2. Final Report of RTCA Task Force 3 Free Flight Implementation. RTCA, Inc., Washington, D.C., Oct. 26, 1995. Available from RTCA at (202) 833-9339.

3. Gibbs, W. W.: Free for All Flights-The FAA Plans a Revolution in Air-Traffic Control. Sci. Amer., vol. 273, no. 6, Dec. 1995, pp. 34-36.

4. Benjamin, Stanley G.; Brundage, Kevin J.; and Morone, Lauren L.: Implementation of the Rapid Update Cycle, Part I: Analysis/Model Description. NOAA/NWS Technical Proceedings Bulletin no. 416. (Also available at http://www.fsl.noaa.gov/frd-bin/tpbruc.cgi.)

5. Devenyi, D.; and Schlatter, T. W.: Statistical Properties of Three-Hour Prediction Errors Derived from the Mesoscale Analysis and Prediction System, Monthly Weather Review, vol. 122, June 1994, pp 1263-1280.
6. Erzberger, Heinz; and Nedell, William: Design of Automated System for Management of Arrival Traffic. NASA TM-102201, June 1989.

7. Ballin, Mark G.; and Erzberger, Heinz: An Analysis of Landing Rates and Separations at the Dallas/Fort Worth International Airport. NASA TM-110397, July 1996.

8. Jardin, Mark R.; and Erzberger, Heinz: Atmospheric Data Acquisition and Interpolation for Enhanced Trajectory-Prediction Accuracy in the Center-TRACON Automation System. Presented at the 34th Aerospace Sciences Meeting and Exhibit, Reno, Nev., Jan. 15-18, 1996.

9. Press, William H.; Teukolsky, Saul A.; Vetterling, William T.; and Flannery, Brian P.: Numerical Recipes in C: The Art of Scientific Computing. Second ed., Cambridge University Press, 1992.

10. Thomas, G. B.; and Finney, R. L.: Calculus and Analytic Geometry. Fifth ed., Addison-Wesley Publishing Co., 1979, p. 430. 
. 
Public reporting burden for this collection of information is estimated to average 1 hour per response, including the time lor reviewing instructions, searching existing data sources, gathering and maintaining the data needed, and completing and reviewing the collection of information. Send comments regarding this burden estimate or any other aspect of this collection of information, including suggestions for reducing this burden, to Washington Headquarters Services, Directorate lor intormation Operations and Reports. 1215 Jefferson Davis Highway, Suite 1204, Arlington, VA 22202-4302, and to the Office of Management and Budget, Paperwork Reduction Project (0704-0188), Washington, OC 20503.

\begin{tabular}{|l|l|l|}
\hline 1. AGENCY USE ONLY (Leave blank) & $\begin{array}{l}\text { 2. REPOAT DATE } \\
\text { October } 1996\end{array}$ & $\begin{array}{l}\text { 3. REPORT TYPE AND DATES COVERED } \\
\text { Technical Memorandum }\end{array}$ \\
\hline
\end{tabular}

4. TITLE AND SUBTITLE

5. FUNDING NUMBERS

Conflict Probability Estimation for Free Flight

6. AUTHOR(S)

$505-64-13$

Russell A. Paielli and Heinz Erzberger

7. PERFoRMING ORgANIZATION NAME(S) AND ADDRESS(ES)

Ames Research Center

Moffett Field, CA 94035-1000

A-962310

9. SPONSORING/MONITORING AGENCY NAME(S) AND AODRESS(ES)

10. SPONSORING/MONITORING AGENCY REPORT NUMBER

National Aeronautics and Space Administration

Washington, DC 20546-0001

NASA TM-110411

11. SUPPLEMENTARY NOTES

Point of Contact: Russell A. Paielli, Ames Research Center, MS 262-3, Moffett Field, CA 94035-1000 (415) 604-5454

12a. DISTRIBUTION/AVAILABILITY STATEMENT

12b. DISTRIBUTION CODE

Unclassified-Unlimited

Subject Category - 03

\section{ABSTRACT (Maximum 200 words)}

The safety and efficiency of free flight will benefit from automated conflict prediction and resolution advisories. Conflict prediction is based on trajectory prediction and is less certain the farther in advance the prediction, however. An estimate is therefore needed of the probability that a conflict will occur, given a pair of predicted trajectories and their levels of uncertainty. A method is developed in this paper to estimate that conflict probability. The trajectory prediction errors are modeled as normally distributed, and the two error covariances for an aircraft pair are combined into a single equivalent covariance of the relative position. A coordinate transformation is then used to derive an analytical solution. Numerical examples and Monte Carlo validation are presented.

14. SUBJECT TERMS

Free flight, Air traffic control, Air traffic management

15. NUMBER OF PAGES 15

16. PRICE CODE

$\mathrm{A03}$

17. SECURITY CLASSIFICATION

OF REPORT

Unclassified

18. SECURITY CLASSIFICATION OF THIS PAGE

Unclassified
19. SECURITY CLASSIFICATION OF ABSTRACT 